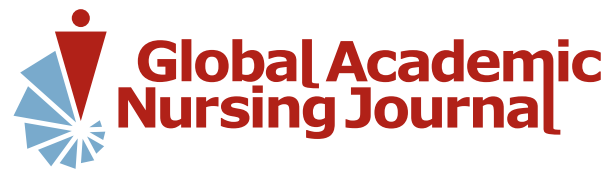

RELATO DE EXPERIÊNCIA

\section{Vivência de enfermeiros em unidade de terapia intensiva destinada a pacientes com COVID-19: relato de experiência}

\author{
Experience of nurses in an intensive care unit for patients with COVID-19: experience report
}

\author{
Experiencia de enfermeras en una unidad de cuidados intensivos para pacientes con COVID-19: informe de \\ experiencia
}

\section{Eva Natalina Ferreira Costa ${ }^{1}$ ORCID: 0000-0003-2673-6967 Ivo da Silva Soares ${ }^{2}$ ORCID: 0000-0001-7163-3073 Flávia Martins Branco ${ }^{1}$ ORCID: 0000-0003-3484-8555 Dayane Martins da Silva Campos $^{1}$ ORCID: 0000-0002-0193-4417 Ana Paula Soares Conceição Benjamin ${ }^{3}$ ORCID: 0000-0002-1510-7439 \\ Ana Cristina de Rodrigues ${ }^{3}$ ORCID: 0000-0001-5254-3515 \\ Adriane da Cunha Aragão Rios Fagundes 4 ORCID: 0000-0003-3041-598X}

${ }^{1}$ Universidade Federal do Estado do Rio de Janeiro. Rio de Janeiro, Brasil. ${ }^{2}$ Centro Universitário IBMR. Rio de Janeiro, Brasil.

${ }^{3}$ Universidade Estácio de Sá. Rio de Janeiro, Brasil.

${ }^{4}$ Faculdades Integradas de Ariquemes. Piauí, Brasil.

\section{Como citar este artigo:}

Costa ENF, Soares IS, Branco FM, Campos DMS, Benjamin APSC, Rodrigues AC, Fagundes ACAR. Vivência de enfermeiros em unidade de terapia intensiva destinada a pacientes com COVID-19: relato de experiência. Glob Acad Nurs. 2021;2(3):e153. https://dx.doi.org/10.5935/26755602.20200153

Autor correspondente: Eva Natalina Ferreira Costa E-mail: evacostaferreira@gmail.com

Editor Chefe: Caroliny dos Santos Guimarães da Fonseca Editor Executivo: Kátia dos Santos Armada de Oliveira

Submissão: 23-07-2021

Aprovação: 30-08-2021

\section{Resumo}

Objetivou-se relatar a atuação de enfermeiros de Unidades de Terapia Intensiva Adulto neste momento de pandemia. Trata-se de um relato de experiência, do tipo descritivo, com abordagem qualitativa, baseado na experiência de enfermeiros, no atual momento de pandemia, em relação ao tempo, a pesquisa delimita-se a partir das práticas e assistência destes profissionais em Unidade de Terapia Intensiva exclusiva para pacientes diagnosticados com COVID-19, período de fevereiro a julho do ano de 2021. A educação continuada e permanente em saúde é imprescindível para qualidade da assistência prestada no atendimento ao paciente com SARS-CoV-2. Manter entre as equipes multiprofissionais uma relação de comunicação é muito importante diante do cenário experienciado. O cenário pandêmico tem um marco de grandes desafios para toda população, referente aos profissionais de enfermagem, as ações de cuidado, das práticas assistenciais, treinamentos, remodelação da gestão de trabalho. Contudo é notório a preparação dos profissionais com conhecimentos sobre: a nova doença COVID-19, pacientes críticos e o uso das novas tecnologias como também proporcionar um local de trabalho adequado.

Descritores: Assistência de Enfermagem; Enfermagem; Pandemias; Infecções por Coronavírus; Unidade de Terapia Intensiva.

\begin{abstract}
The aim was to report the performance of nurses in Adult Intensive Care Units at this time of pandemic. This is an experience report, of a descriptive type, with a qualitative approach, based on the experience of nurses, in the current moment of pandemic, in relation to time, the research is delimited from the practices and assistance of these professionals in a Health Unit. Exclusive Intensive Care for patients diagnosed with COVID-19, from February to July 2021. Continuing and continuing education in health is essential for the quality of care provided in the care of patients with SARS-CoV-2. Maintaining a communication relationship between the multidisciplinary teams is very important in view of the experienced scenario. The pandemic scenario has a framework of great challenges for the entire population, referring to nursing professionals, care actions, care practices, training, remodeling of work management. However, the preparation of professionals with knowledge about the new disease COVID-19, critically ill patients and the use of new technologies is notorious, as well as providing an adequate workplace
\end{abstract}

Descriptors: Nursing Care; Nursing; Pandemic; Coronavirus Infections; Intensive Care Unit.

\section{Resumén}

El objetivo fue reportar el desempeño de las enfermeras en las Unidades de Cuidados Intensivos de Adultos en este momento de pandemia. Se trata de un relato de experiencia, de tipo descriptivo, con abordaje cualitativo, basado en la experiencia de enfermeras, en el momento actual de pandemia, en relación al tiempo, la investigación se delimita desde las prácticas y asistencia de estos profesionales en un ámbito de la Salud. Unidad. Cuidados intensivos exclusivos para pacientes diagnosticados de COVID-19, de febrero a julio de 2021. La educación continua y continua en salud es fundamental para la calidad de la atención brindada en la atención de los pacientes con SARS-CoV-2. Mantener una relación de comunicación entre los equipos multidisciplinares es muy importante a la vista del escenario vivido. El escenario pandémico tiene un marco de grandes desafíos para toda la población, referidos a profesionales de enfermería, acciones de cuidado, prácticas de cuidado, capacitación, remodelación de la gestión del trabajo. Sin embargo, es notoria la preparación de profesionales con conocimientos sobre: la nueva enfermedad COVID-19, pacientes críticos y el uso de nuevas tecnologías, además de brindar un lugar de trabajo adecuado.

Descriptores: Atención de Enfermería; Enfermería; Pandemia; Infecciones por Coronavirus; Unidades de Cuidados Intensivos. 


\section{Introdução}

O SARS-CoV-2 é um vírus que causa a doença (Coronavírus Disease 2019 ou conhecida também por COVID-19), que após o surgimento do primeiro caso em dezembro de 2019 na cidade de Wuhan na China, gerou uma emergência em saúde pública global, em 2020 no mês de junho havia mais de 6,4 milhões de casos confirmados e 385.000 mil mortes em todo o mundo ${ }^{1}$. Os sinais clínicos da doença envolvem febre, fadiga, tosse seca, e sintomas como gastrointestinais e dificuldades de respirar ${ }^{2}$.

No Brasil em meados de janeiro de 2020 foi acionado o Centro de Operações de Emergência (COE) em Saúde Pública para elaboração de estudos e protocolos sobre a doença, o novo coronavírus (COVID-19) que se espalhavam pelo mundo. A vigilância epidemiológica de infeção humana pelo SARS CoV-2 será construída e consolidada pela OMS, com referências trazidas dos países com evidências científicas publicadas ${ }^{3}$.

Com a descoberta deste então, inúmeros pacientes infectados pelo SARS-CoV-2 apresentaram uma síndrome respiratória aguda grave (SRAG) e precisaram de internações em Unidades de Terapia Intensiva (UTI). Desta forma a pandemia tornou se um momento desafiador para todos os Sistemas de Saúde Mundiais, devido à grande demanda por leitos nas Unidades de Terapias Intensivas e profissionais de saúde como enfermagem ${ }^{4}$.

No Brasil as desigualdades sociais e de saúde foram elementos para que algumas regiões no país devido à falta de leitos de Unidade de Terapia Intensiva e de recursos humanos, possuíssem taxas de mortalidade maiores que a média nacional. No Sistema Único de Saúde mais de $72 \%$ das regiões tinham uma quantidade de leitos menor que o recomendado pelos Órgãos Sanitários. Ocorrendo superlotação e sobrecarga das Unidades de Saúde. A propagação do vírus no país está diretamente relacionada a cobertura da rede de atenção à saúde, assim como o arsenal médico assistencial disponível nestas regiões ${ }^{5}$.

Pesquisas realizadas em Unidades de Terapias Intensivas mostrou se uma taxa de mortalidade de doentes com COVID-19 de $50 \%$ e mais de $75 \%$ precisam de ventilação mecânica invasiva, com um tempo maior que duas semanas, certificando a necessidade de equipes de enfermagem capacitadas para atuar nestes cenários, em que a sobrecarga de trabalho e o estresse são excessivos ${ }^{6}$.

Os cuidados realizados aos pacientes pela equipe de enfermagem na unidade de terapia intensiva, requer conhecimentos, habilidades e atitudes, pois o manejo é diferente e desafiador e precisam de capacitações técnicos cientifica consecutivas para o desenvolvimento profissional, pois as técnicas realizadas trazem desafios para o enfermeiro e sua equipe ${ }^{6,7}$.

Frente a este momento de pandemia, os enfermeiros e enfrentam desafios relevantes no que concerne o cuidado e qualidade da assistência de enfermagem nas unidades de Terapia Intensiva. Os enfermeiros dos países China e Itália mostraram com suas experiências que os maiores obstáculos na Assistência aos indivíduos com a doença COVID-19 se deram no âmbito da estruturação de equipes treinadas e capacitadas, dimensionamento de pessoal, gerenciamento de insumos, equipamentos e o cuidado da saúde destes profissionais ${ }^{8-9}$.

Neste cenário de pandemia mundial, as medidas educativas são fundamentais, principalmente se tratando de uma doença desconhecida. As medidas preventivas de proteções individuais e coletivas para os profissionais são extremamente relevantes para segurança e proteção destes profissionais. Estar na linha de frente é desafiador, causa sentimentos de medos, ansiedades e angústia por perdas dos pacientes.

O exposto estudo tem como objetivo relatar a atuação de enfermeiros da Unidade de Terapia Intensiva Adulto, neste momento de pandemia do novo coronavírus.

\section{Metodologia}

O presente estudo trata-se de um relato de experiência, do tipo descritivo, com abordagem qualitativa. Esse tipo de pesquisa possibilita a disseminação de conteúdo através de vivências dos próprios autores, transmitindo então, conhecimento cientifico ${ }^{10}$.

O estudo foi baseado através da experiência de profissionais enfermeiros, no atual estado de pandemia, construído enquanto atuantes como enfermeiros em Unidades de Terapia Intensiva Adulto (UTI-Adulto) de hospitais públicos da Cidade do Rio de Janeiro.

O setor da localidade escolhida, é destinado para pacientes diagnosticados com o COVID-19, delimitando-se dentro do período de fevereiro a julho do ano de 2021.

Dentre todas as vivências, também foi descrito os sentimentos e desafios enfrentados por esses profissionais, durante esse período de atuação, destacando como a pandemia afetou a rotina de trabalho e a saúde mental dos enfermeiros.

\section{Relato de Experiência}

Durante a atuação foi possível perceber, inicialmente, que a educação continuada e permanente em saúde é imprescindível para qualidade da assistência prestada no atendimento ao paciente com SARS-CoV-2, tendo em vista uma crescente demanda por profissionais qualificados para atuação nas UTIs.

Outro fator da vivência é a manutenção, entre as equipes multiprofissionais, de uma relação de comunicação, sendo extremamente relevante diante do cenário experienciado, pois ocorreu um grande aumento do número de casos, que iniciaram em maio de 2021. Sendo assim, os hospitais destinaram unidades exclusivas para esses pacientes, sendo ofertado treinamentos para os profissionais, como por exemplo, sobre padronização do uso correto dos equipamentos de proteção individual (EPI). Diante disso, percebeu-se a gravidade desses pacientes e como eles carecem de um quantitativo de profissionais de enfermagem com toda a equipe qualificada.

Nos primeiros meses de atuação, os pacientes já chegavam entubados na UTI, entravam pela emergência e quando apresentavam gravidades clínicas eram transferidos para unidade de pacientes graves.

A comunicação com os familiares era realizada por uma equipe de criada pelos Gabinetes de crises dos 
hospitais. Neste período nos deparamos com a gravidade clínica dos pacientes, onde com um tempo maior de internação, apresentavam pioras constantes no comprometimento sistêmico pela doença e apresentando uma recuperação muito lenta. Sendo assim, experienciamos a aflição e um sentimento de inutilidade diante deste cenário com os cuidados aos indivíduos infectados pelo novo coronavírus.

Durante este período vale destacar que o uso das videoconferências foi utilizado para reuniões, aulas, palestras para capacitação dos profissionais com a finalidade de aperfeiçoamento nas práticas assistenciais. Possibilitando o treinamento dos novos profissionais contratados devido a demanda no dimensionamento de pessoal. Muitos destes não tinham experiência em terapia intensiva. Em consequência desta alta demanda nos serviços de saúde, os enfermeiros indubitavelmente têm uma atribuição primordial no enfrentamento desta pandemia.

A enfermagem brasileira vive diante de muitos desafios, nesta pandemia evidenciaram aspectos relevantes como, baixos salários e condições inadequadas de trabalho, dimensionamento de pessoal diminuído, sendo assim para que tenhamos uma assistência baseada em evidência, faz se necessário o estudo acerca da patologia e os cuidados com a prevenção da doença e o uso das tecnologias.

\section{Discussão}

No Brasil, a pandemia mostra adversidades estruturais presentes no Sistema de Saúde, como falta de investimentos em pesquisas, no próprio sistema, déficit de profissionais qualificados e a desarticulação entre os níveis de atenção à saúde ${ }^{11}$. O cuidado aos pacientes graves esbarra com uma falta cada vez maior de profissionais enfermeiros capacitados que possam atuar nas Unidades de Terapia intensiva ${ }^{12}$.

Este cenário pandêmico simboliza na saúde do século XXI um grande desafio até o presente momento. A COVID-19 detém repercussões relevantes no Sistema de Saúde Nacional, notadamente no que refere a criação e manutenção de leitos em terapia intensiva a compra de ventiladores mecânicos, organização dos serviços e de pesquisas quanto a descoberta de vacinas e terapêuticas manipuladas nos doentes ${ }^{13}$.

O cenário atual mostra o funcionamento dos hospitais com superlotação com dimensionamento de pessoal reduzido, falta de insumos, profissionais sobrecarregados, fisicamente e mentalmente. Diante disso aponta se a necessidade de viabilizar assistência a categoria que intermedeia a história colaborando e executando de forma infindável para o cuidado com aqueles que estão doentes ${ }^{13}$.

O trabalho das equipes de enfermagem obteve atuais paradigmas, pois além da assistência, transformaram em um canal de comunicação entre pacientes e familiares, compartilhando as aflições experienciadas juntos a perda de seus colegas de trabalho. As equipes têm um papel relevante, pois administram e contribuem com o cuidado, trabalhando para proporcionar a recuperação dos indivíduos e dentro do possível fornecendo tranquilidade aos familiares. Muitos profissionais ficaram longes de seus familiares como forma de prevenção ${ }^{14}$.

As complicações mais comuns da doença COVID-19 apresentadas nos pacientes é a síndrome da Angústia respiratória aguda (SDRA), somando as outras desordens como injuria renal, lesões cardíacas e alveolares e infecções secundárias. No que concerne a cuidado de enfermagem a estas condições clínicas, o uso da terapia renal substitutiva (hemodiálise) e a utilização da posição prona tem sido um dos procedimentos mais aplicados tanto nas unidades de terapias intensivas quanto nas Unidades de internação clínicas e pacientes graves, com o intuído de diminuição da mortalidade e conforto para estes pacientes com coronavírus $^{15}$.

A maioria dos pacientes necessitavam de pronação, nesta manobra, o enfermeiro tem um papel essencial na aplicação da prática clínica e no gerenciamento do processo e implementação da técnica. No Brasil temos uma vasta literatura sobre o processo de aplicabilidade por meio de checklist sobre a manobra prona. A padronização da técnica é muito importante para atualidade, vale lembrar que a manobra possui problemas relevantes como lesões por pressão, por dispositivos médicos, pneumonia associada a ventilação mecânica e extubação acidental ${ }^{16}$.

As implicações da pandemia na vida dos profissionais de enfermagem do Brasil ainda são desconhecidas. Portanto, estudos produzidos com enfermeiros e técnicos e auxiliares de enfermagem de outros países como na China, com doenças provocadas por vírus parecidos como SARS-CoV-2 apresentaram repercussões negativas na saúde destes indivíduos, evidenciando infecção pelo vírus, Síndrome de Burnout; estresse provocado pela pressão das organizações e da sociedade, obstáculos éticos na realização dos procedimentos, transtornos de ansiedade, depressivos, estresse pós traumático; automedicação em excesso devido o cansaço ou adoecimento mental: medo e insegurança em infectar suas famílias. Além da vivência com altos números de óbitos ${ }^{17}$.

\section{Saúde Mental dos Profissionais de Enfermagem da Unidade de Terapia Intensiva}

Confirmando os resultados sobre os sentimentos vivenciados pelos enfermeiros, a pesquisa de Caldas $^{18}$ evidencia que os profissionais da área da saúde enfrentaram medo, preocupação constante, estresse pela situação e ainda desenvolvendo problemas de saúde mental, como ansiedade, depressão e insônia relacionada aos mesmos.

$O$ trabalho da equipe de enfermagem na assistência aos pacientes críticos é causador de sofrimentos psíquicos. No cenário que experienciamos de isolamento social é de muita pressão nos serviços de saúde impulsionando sensações de abandono, desprazeres, tristezas e repercussões orgânicas como distúrbio do sono e apetite ${ }^{19}$.

A sobrecarga de trabalho do enfermeiro e o comprometimento técnico, pode colaborar para o desequilíbrio emocional destes profissionais, o surgimento da pandemia de COVID-19 corroborou com esta situação, que originou se em drásticas mudanças nas relações de 
trabalho e na vida social e fez com que estes profissionais ficassem mais vulneráveis a este tormento ${ }^{18}$. Fatores importantes como falta de equipamentos de proteção individual, treinamentos, testes diagnósticos falta de dimensionamento de pessoal adequado, informações e conhecimento relacionados a nova doença, aumento do número de profissionais de enfermagem infectados e a desvalorização de toda categoria ${ }^{20}$.

A equipe de enfermagem é o suporte psicológico, cujo indivíduo cria um vínculo no período em que precisa de atenção à saúde, no entanto neste momento da pandemia esta equipe não pode realizar esta atividade de feitio de forma adequada pelo motivo do impacto causado em sua saúde emocional. A Organização Mundial de Saúde, Conselho Federal de Enfermagem e os Órgãos Regionais identificando essa necessidade psicológica proveniente dos trabalhadores dos serviços de saúde, organizaram intervenções e cuidados psicológicos para os profissionais ${ }^{21}$.

Além disso, defronta constantemente com um acúmulo de informações corrompidas advindas dos meios de comunicações como mídias sociais, que enfraquece a conduta da população em relação a responsabilidade com a própria saúde ${ }^{22}$. Em contrapartida a isso, em uma outra pesquisa, é possível visualizar outro ponto de vista sobre as redes sociais, informando que estas possuem a capacidade e facilidade de repassar informações em tempo real para toda a população, ou seja, as mídias possuem pontos negativos e positivos e que depende apenas da forma que será utilizado e quais fontes estão sendo lidas ${ }^{23}$.
Percebe-se então, que os profissionais de saúde enfermagem passam por desafios neste período de pandemia pois vivenciam escassez de recursos humanos, sobrecarga de trabalho, falta de equipamentos de proteção individual insumos em geral, diante de tudo isso ainda enfrentam a incerteza de tratamentos realizados e o medo, apreensão da coordenação da própria saúde e de seus pacientes e familiares ${ }^{24}$.

\section{Conclusão}

Conclui-se que o cenário pandêmico tem um marco de grandes desafios para toda população, destacando-se a equipe de enfermagem, neste caso o enfermeiro, pois estão diretamente relacionados com as ações de cuidado das práticas assistenciais, treinamentos, remodelação da gestão de trabalho.

Contudo é notório a necessidade preparação dos profissionais com conhecimentos sobre: a nova doença COVID-19, pacientes críticos e o uso das novas tecnologias como também proporcionar um local de trabalho adequado, mas que além disso, preparar os profissionais enfermeiros no quesito psicológico para tua atuação.

Como recomendação para trabalhos futuros, cita-se a realização de estudos voltados para todas as áreas de saúde, realizando comparação para verificar se há diferença no impacto mental entre as profissões, ou seja, entender as experiências de diferentes áreas e como estas reagem ao estado de saúde atual frente à COVID-19.

\section{Referências}

1. Tabah A, et al. Personal protective equipment and intensive care unit healthcare worker safety in the COVID-19 era (PPE-SAFE): An international survey. J Crit Care. 2020;59:70-75. DOI: 10.1016/j.jcrc.2020.06.005

2. Guo YR, et al. The origin, transmission and clinical therapies on coronavírus disease 2019(COVID-19) impacted on de humanization of ICU patients update on the Status. Mil Med Res. 2020;7(11). https://dx.doi.org/10.1186\%2Fs40779-020-00240-0

3. Ministério da Saúde (BR). Protocolo de manejo clínico da COVID-19 na Atenção Especializada. Departamento de Atenção Hospitalar Domiciliar e de Urgência. 1. Ed. Brasília (DF): MS; 2020.

4. Raurell-Torreda M. Gestion de los equipos de enfermeria de uci durante la pandemia COVID-19. Enfermaria Intensiva. 2020;31(2):49. https://dx.doi.org/10.1016\%2Fj.enfi.2020.04.001

5. Rache B, et al. Necessidades de Infraestrutura do SUS em preparo COVID-19 leitos de UTI respiradores e ocupação hospitalar. São Paulo (SP): Instituto de Estudos para Políticas de Saúde; 2020.

6. Bhatraju PK, et al. COVID-19: in Critically, III Patients in the Seatle Region - Case Series. N Engl J Med. 2020;382(21):2020-2021. DOI: 10.1056/NEJMoa2004500

7. Associação de Medicina Intensiva Brasileira (AMIB). Diretrizes e Recomendações [Internet]. 2020 [acesso em 02 jun 2021]. Disponível em: https://www.amib.org.br/covid-19/diretrizes-e-recomendacões

8. Kang L, et al. Impact on mental health and perceptions of psychological care among medical and nursing statf in Wuhan during the 2019 novel coronavírus disease outbreak: A cross- sectional study. Brain Behav Immun. 2020;87:11-17. DOI: 10.1016/j.bbi.2020.03.028

9. Sharma SK, et al. Clinical nursing care guidance for management of patient with COVID -19. J Park Med Assoc. 2020;70(Suppl 3-5):SI18S123. https://doi.org/10.5455/jpma.29

10. Grollmus NS, Tarrés JP. Relatos metodológicos: difractando experiências narrativas de investigación. Fórum Qualitative Social Research. $2015 ; 16(2)$.

11. Brito SBP, et al. Pandemia da COVID-19: O maior desafio do século XXI. Vigilância Sanitária em Debate: Sociedade e Tecnologia [Internet]. 2020 [acesso em 28 jun 2021];8(2):54-63. Disponível em:

https://visaemdebate.incqs.fiocruz.br/index.php/visaemdebate/article/view/1531

12. Nunes MR. A atuação do enfermeiro em unidade de terapia intensiva na pandemia de COVID-19: relato de experiência. Revista Eletrônica Acervo Saúde. 2020;12(11):01-06. http://dx.doi.org/10.25248/reas.e4935.2020

13. Luz EMF, et al. Repercussões da COVID-19 na saúde mental dos trabalhadores de enfermagem. Revista de Enfermagem do Centro-Oeste Mineiro [Internet]. 2020 [acesso em 29 mar 2021];10:e3824. Disponível em: http://seer.ufsj.edu.br/index.php/recom/article/view/3824/2426 
14. Tanaka AKSR, et al. A importância da equipe de enfermagem em tempos de pandemia. Blog LE-VI@enf [Internet]. 2021 [20 abr 2021 ]. Disponível em: https://www.ufrgs.br/levi/equipedeenfermagem-na-pandemia/\#page-content

15. Fernandes CA, et al. Desafios e recomendações para o cuidado intensivo de adultos críticos com doença de coronavírus 2019 (COVID-19). HRJ. 2020;(1):21-47. https://dx.doi.org/10.51723/hrj.v1i1.20

16. Oliveira VM, et al. Checklist da prona segura: construção e implementação de uma ferramenta para realização da manobra de prona. Revista Brasileira de terapia intensiva. 2017;29(2):131-141. https://doi.org/10.5935/0103-507X.20170023

17. Souza LPS, Souza AG. Enfermagem Brasileira na linha de frente contra o Novo Coronavírus: Quem cuidará de quem cuida? J. nurs. Health [Internet]. 2020 [acesso em 08 jul 2021];10(Esp.). Disponível em: https://docs.bvsalud.org/biblioref/2020/05/1095606/1-enfermagembrasileira-na-linha-de-frente-contra-o-novo-coron_ygPksqt.pdf

18. Caldas GRF, et al. Novo Coronavírus: Impacto na saúde mental dos profissionais de saúde. RSD. 2021;10(5). http://dx.doi.org/10.33448/rsd-v10i5.15011

19. Nunes MR. A atuação do enfermeiro em unidade de terapia intensiva na pandemia de COVID-19: relato de experiência. Revista Eletrônica Acervo Saúde. 2020;12(11):1-6. http://dx.doi.org/10.25248/reas.e4935.2020

20. Silva AF, Robazzi MLCC. Alterações mentais em trabalhadores de unidades de terapia intensiva. SMAD, Rev. Eletrônica Saúde Mental Álcool Drog. 2019;15(3):1-10. http://dx.doi.org/10.11606/issn.1806-6976.smad.2019.151483

21. Lentz GNS, Batista EA, Zanon J, Silva LF. As implicações emocionais na saúde dos enfermeiros durante a pandemia do SARS-CoV-2. Glob Acad Nurs. 2021;2(1):e80. https://dx.doi.org/10.5935/2675-5602.20200080

22. Ramos-Toescher, AM, et al. Saúde mental de profissionais de enfermagem durante a pandemia de COVID-19: recursos de apoio. Escola Anna Nery. 2020;24(Spe):e20200276. https://doi.org/10.1590/2177-9465-EAN-2020-0276

23. Xavier F, Olenscki JRW, Acosta AL, Sallum MAM, Saraiva AM. Análise de redes sociais como estratégia de apoio à vigilância em saúde durante a COVID-19. Pandemia pela COVID-19. Estud. av. 2020;34(99). https://doi.org/10.1590/s0103-4014.2020.3499.016

24. Gaino LV, Souza J, Cirineu CT, Tulimosky TD. O conceito de saúde mental para profissionais de saúde: um estudo transversal e qualitativo. SMAD. 2018;2:108-16. http://dx.doi.org/10.11606/issn.1806-6976.smad.2018.149449 